\title{
Crossing time in the dissipative Landau-Zener quantum dynamics
}

\author{
P. Nalbach ${ }^{\mathrm{a}}$ (iD \\ Fachbereich Wirtschaft und Informationstechnik, Westfälische Hochschule, Münsterstrasse 265, 46397 Bocholt, Germany
}

Received 13 December 2021 / Accepted 22 February 2022 / Published online 5 March 2022 (C) The Author(s) 2022

\begin{abstract}
We study the dynamics of a quantum two-state system driven through an avoided crossing under the influence of a super-Ohmic environment. We determine the Landau-Zener probability employing the numerical exact quasi-adiabatic path integral and a Markovian weak coupling approach. Increasing the driving time in the numerical protocol, we find converged results which shows that super-Ohmic environments only influence the Landau Zener probability within a finite crossing time window. This crossing time is qualitatively determined by the environmental cut-off energy. At weak coupling, we show that the Markovian weak coupling approach provides an accurate description. Since pure dephasing of a super-Ohmic bath is non-Markovian, this highlights that pure dephasing hardly influences the LandauZener probability. The finite crossing time window, thus, results from the suppression of relaxation once the energy splitting exceeds the environmental cut-off energy.
\end{abstract}

\section{Introduction}

The transition dynamics of a driven quantum system in the vicinity of avoided crossings of its energy levels [1-4] is at the heart of various very different physical problems. Examples are the dynamics of chemical reactions [5], the spin reversal dynamics in molecular nanomagnets [6], the non-equilibrium dynamics of glasses at low temperatures [7-10], the dynamics of solid state artificial atoms $[11,12]$, and transfer of information between distant electron spin qubits [13-19]. Typically, quantum systems are also influenced by their environments which exert fluctuating forces on them resulting in decoherence and relaxation $[20,21]$. The according relaxation or dephasing times then compete with the time scale of driving and significantly influence the Landau-Zener switching dynamics $[22,23]$.

Dissipative quantum dynamics at an avoided crossing is widely studied [22-34] in the simplified Landau-Zener model, i.e., for a quantum two-state system (TSS) linearly driven through an avoided crossing [1-4]. Typically, the Landau-Zener probability $P_{\text {LZ }}$ to end up in the ground state when the system started there or, alternatively, the excited-state survival probability $P_{\mathrm{ES}}$ to end up in the excited state when the system started from the excited state is studied. While for non-adiabatic driving, an environment hardly influences the Landau-Zener dynamics, for adiabatic driving, a crossover is observed where at finite temperature relaxation strongly influences the Landau-Zener dynamics $[22,23,30]$.

\footnotetext{
a e-mail: Peter.Nalbach@w-hs.de (corresponding author)
}

The driving protocol of the Landau-Zener model runs for an infinite time, but the tunnel coupling between the two states influences the dynamics only, while it exceeds their energy splitting restricting the dynamics to a Landau-Zener crossing time window during which the avoided crossing takes place. This finally allows to employ the theoretical results from the simplified Landau-Zener model for the description of real systems where driving is always finite. Relaxation results from energy exchange between system and environment. Thus, with increasing energy splitting away from the avoided crossing relaxation should influence the dynamics until the energy splitting exceeds roughly the maximal excitation energy of the environment. Surprisingly, a longitudinal Ohmic bath influences the Landau-Zener probability only within a time window similar to the Landau-Zener crossing time [22,23], i.e., as long as the tunnel coupling exceeds the energy splitting. A transversal Ohmic bath, in contrast, influences the dynamics in the much wider time window around the avoided crossing [30] determined roughly by the regime where the energy splitting does not exceed the maximal excitation energy of the environment. Both cases can be understood within a Markovian weak coupling approach $[35,36]$ where time-dependent effective relaxation rates can be determined. These relaxation rates are strongly suppressed outside the time windows in which the environment influences the dynamics $[23,30]$. In contrast, pure dephasing rates for the longitudinal Ohmic environment are not suppressed, but nevertheless seem not to affect the studied LandauZener probability $P_{\mathrm{LZ}}$ nor the excited-state survival probability $P_{\mathrm{ES}}$. 
The crossing time window, in which dissipation is relevant, strongly depends on the environment. We, therefore, extend the studies to a super-Ohmic environment. The influence of a super-Ohmic environment on the Landau-Zener dynamics has not been studied in detail yet. Whitney et al. [31] studied super-Ohmic influence on the coherent oscillations in the dynamics after the avoided crossing. The questions to answer are whether the influence of a super-Ohmic environment is also restricted to a crossing time window and, if so, what determines this crossing time window. While a Markovian weak coupling approach allows to determine the effective relaxation rates, pure dephasing in a superOhmic environment is non-Markovian [20] and, thus, cannot be described. Thus, it is also unclear whether the Markovian weak coupling approach $[35,36]$ is able to describe the Landau-Zener probability $P_{\mathrm{LZ}}$ and the excited-state survival probability $P_{\mathrm{ES}}$ under the influence of a super-Ohmic environment.

We observe that a longitudinal super-Ohmic bath shows a crossing time window similar to a transversal Ohmic bath. A transverse super Ohmic bath, however, exhibits again a much wider crossing time window which renders a numerical exact simulation even at very small system-bath couplings' challenging. We determine the Landau-Zener and the excited-state survival probabilities numerically exact employing the quasi-adiabatic path integral (QUAPI) $[22,37,38,40]$. We show that at weak coupling, the adiabatic Markovian non-equilibrium Bloch equations (NEQBs) [35,36] indeed can reliably simulate both.

In the next three sections, we introduce our model, our observables, and the used methods. In Sect. 5, we determine the Landau-Zener and the excited-state survival probability as function of drive parameters for various temperatures and system-bath couplings. Comparing numerically exact and a Markovian weak coupling approach, we establish in which parameter regime the weak coupling approach indeed describes the dynamics. This allows then to conclude that pure dephasing is not relevant and to discuss the crossing time windows in which relaxation actively influences the dynamics. Finally, we end with a conclusion.

\section{Model}

A quantum two-level system (TSS) driven through an avoided crossing is modelled by an Hamiltonian

$$
H_{S}(t)=\frac{\Delta}{2} \sigma_{x}+\frac{\epsilon(t)}{2} \sigma_{z}
$$

with Pauli matrices $\sigma_{i}$, driving field $\epsilon(t)$ and tunnel coupling $\Delta$ between the two eigenstates of the system at $t= \pm \infty$. We discuss a linear driving protocol, i.e., $\epsilon(t)=v t$ with sweep or drive speed $v$ and the avoided crossing at $t=0$.

We model environmental noise acting on the TSS in a system-bath approach $[20,21]$ by coupling a bath of harmonic oscillators, i.e. $H_{B}=\sum_{k} \omega_{k} b_{k}^{\dagger} b_{k}$, bilinearly to the TSS resulting in a total Hamiltonian [20,21]

$$
H(t)=H_{S}(t)-\frac{\sigma_{z} \cos \Theta+\sigma_{x} \sin \Theta}{2} \hat{B}+H_{B}
$$

and $\hat{B}=\sum_{k} \lambda_{k}\left(b_{k}+b_{k}^{\dagger}\right)$ with bosonic annihilation $b_{k}$ and creation $b_{k}^{\dagger}$ operators. The system-bath coupling is denoted as longitudinal for $\Theta=0$ and transversal for $\Theta=\pi / 2$. The bath influence is captured by a spectral function

$$
G(\omega)=\sum_{k} \lambda_{k}^{2} \delta\left(\omega-\omega_{k}\right)=\frac{\gamma \Delta^{1-s}}{\pi} \omega^{s} \exp \left(-\omega / \omega_{c}\right)
$$

with cut-off frequency $\omega_{c}$, the coupling strength $\gamma$, and the spectral exponent $s$. For $s=1$ the bath is termed Ohmic and for $s>1$ super-Ohmic. In the following, we focus on $s=3$ which reflects, for example, phonons in a $3 \mathrm{D}$ system coupled to an acoustic dipole [9,41-43].

\section{Observables}

In the Landau-Zener protocol, we drive the TSS with $\epsilon(t)=v t$ starting at $t=-\infty$ and ending at $t=\infty$. We determine the Landau-Zener probability $P_{\text {LZ }}$ to end up in the ground state when the system started in the ground state, that is

$$
P_{\mathrm{LZ}}=\operatorname{Tr}\{\hat{g} \rho(t=\infty)\}
$$

with $\hat{g}=\frac{1}{2}\left(\mathbb{1}-\sigma_{z}\right)$ and $\rho(t=\infty)$ the statistical operator at the end of the driving protocol when $\rho(t=-\infty)=\hat{g}$. We also calculate the excited-state survival probability $P_{\text {ES }}$ to end up in the excited state when the system started from the excited state, that is

$$
P_{\mathrm{ES}}=\operatorname{Tr}\{\hat{e} \rho(t=\infty)\}
$$

with $\hat{e}=\frac{1}{2}\left(1+\sigma_{z}\right)$ and $\rho(t=\infty)$ the statistical operator at the end of the driving protocol when $\rho(t=-\infty)=\hat{e}$.

Without environment (indicated by the superscript $(0))$, these probabilities are

$$
P_{\mathrm{LZ}}^{(0)}=P_{\mathrm{ES}}^{(0)}=1-e^{-\frac{\pi \Delta^{2}}{2 v}}
$$

with adiabatic behaviour for $v \ll \Delta^{2}$, diabatic behaviour for $v \gg \Delta^{2}$, and a mixed regime for $v \simeq \Delta^{2}$ $[1-4]$.

\section{Methods}

Including now the coupling to the bath, closed forms for $P_{\mathrm{LZ}}$ and $P_{\mathrm{ES}}$ are not available and we are forced to determine the time-dependent dynamics, i.e., the 
reduced density $\rho(t)$ of the TSS, numerically. The Landau-Zener driving protocol runs from the infinite past to the avoided crossing at $t=0$ to the infinite future. Numerical investigation relies on the fact that the dynamics is determined in the proximity of the avoided crossing. Accordingly, converged results are quickly obtained for protocols running from $-t_{\max } / 2$ to $t_{\max } / 2$ when increasing $t_{\max }$. An extended time window in which relaxation is finite, renders the Landau-Zener dynamics substantially more difficult to study numerically, since with increasing investigation time $t_{\max }$, the numerically accumulated errors increase.

\subsection{Non-equilibrium Bloch equations}

For weak system-bath coupling, a Markovian Redfield type approach is known to describe the LandauZener dynamics correctly $[35,36,44-47]$ for an Ohmic bath. It leads to relaxation and dephasing rates with time dependence governed by the time dependence of the driving field. We employ the non-equilibrium Bloch equations (NEBQs) [36]. Transforming the system [Eq.(1)] to $\widetilde{H}_{S}(t)=R^{\dagger}(t) H_{S}(t) R(t)=E(t) \tau_{x} / 2$ by $R(t)=\exp \left[i \phi(t) \sigma_{y} / 2\right]$ with $\phi(t)=\arctan (\epsilon(t) / \Delta)$ and Pauli matrices $\tau_{i}$ and $E(t)=\sqrt{\Delta^{2}+\epsilon^{2}(t)}$ results in an effective Hamiltonian $\widetilde{H}_{S}(t)+\phi^{\prime}(t) \tau_{y} / 2$ governing the dynamics (besides the coupling to the bath). Introducing the reduced density operator for the system $\rho_{S}(t)=\frac{1}{2}\left(\mathbb{1}-r_{i} \tau_{i}\right)$ and employing the Markovian Redfield type approach leads to NEQBs

$$
\begin{aligned}
& \partial_{t} r_{x}(t)=+\phi^{\prime}(t) r_{z}(t)-\Gamma_{1}(t)\left[r_{x}(t)-r_{x}^{s t}(t)\right] \\
& \partial_{t} r_{y}(t)=-\Gamma_{2}(t) r_{y}(t)-E(t) r_{z}(t) \\
& \partial_{t} r_{z}(t)=+E(t) r_{y}(t)-\Gamma_{2}(t) r_{z}(t)-\phi^{\prime}(t) r_{x}(t)
\end{aligned}
$$

with $r_{x}^{(s t)}(t)=\tanh (\beta E(t) / 2)$ and

$$
\Gamma_{1}[s, \Theta](t)=A_{\Theta}^{2}(t) \frac{\pi}{2} G(E(t)) \operatorname{coth}\left(\frac{\beta E(t)}{2}\right)
$$

with

$$
\begin{aligned}
A_{\Theta}(t) & =u(t) \cos \Theta-v(t) \sin \Theta \\
u(t) & =\cos \varphi(t)=\frac{\Delta}{E(t)} \\
v(t) & =\sin \varphi(t)=\frac{\epsilon(t)}{E(t)} .
\end{aligned}
$$

and

$$
\Gamma_{2}[s, \Theta](t)=\frac{1}{2} \Gamma_{1}[s, \Theta](t)+\Gamma_{d}[s, \Theta](t)
$$

with

$$
\begin{aligned}
\Gamma_{d}[s, \Theta] & =B_{\Theta}^{2}(t) \frac{\pi}{2} \lim _{\omega \rightarrow 0}[G(\omega) \operatorname{coth}(\beta \omega / 2)] \\
& =\left\{\begin{array}{ll}
B_{\Theta}^{2}(t) \gamma k_{\mathrm{B}} T & : s=1 \\
0 & : s>1
\end{array}\right. \text { with }
\end{aligned}
$$

$$
B_{\Theta}(t)=v(t) \cos \Theta+u(t) \sin \Theta
$$

The pure dephasing rate $\Gamma_{d}(t)$ has only for an Ohmic environment a finite Markovian contribution. However, for an asymmetric TSS (which exhibits a finite $B_{\Theta}$ ) in a super-Ohmic bath, the Markovian approximation is not reliable for describing pure dephasing [20].

\subsection{QUAPI}

The quasi-adiabatic path integral (QUAPI) [22,37-40] was used to obtain numerical exact results for the dissipative Landau Zener dynamics. QUAPI is based on a symmetric Trotter splitting of the short-time propagator $\mathcal{K}\left(t_{k+1}, t_{k}\right)$ (describing time evolution over a time slice $\delta t$ ) for the full Hamiltonian $H$. The splitting is by construction exact in the limit $\delta t \rightarrow 0$, but introduces a finite Trotter error for a finite time increment, which has to be eliminated by choosing $\delta t$ small. The QUAPI scheme further employs an approximated Feynman-Vernon influence functional which includes only non-local time correlations between observables in a time window $\tau_{\text {mem }}=k_{\max } \delta t$. To obtain a representation for the Feynman-Vernon influence function, a static system basis is chosen for which the system part of the system-bath coupling operator is diagonal. Timedependence of the system Hamiltonian is fully captured by the system's short-time propagator [39]. Generally, valid results are achieved by finding convergence while increasing $\tau_{\text {mem }}$ but at the same time decreasing $\delta t$ to minimize the Trotter error. For a detailed discussion on how to achieve convergence regarding these two parameters, see $[38,40]$. We focus here the investigation on convergence with respect to the key quantities of the paper: $P_{\mathrm{LZ}}$ and $P_{\mathrm{ES}}$ which reflect additionally the long time limit of the driven dynamics. As discussed below in detail, we observe rather extended crossing time windows in which the bath influences the system dynamic, specifically for the transversal super-Ohmic bath. Then, the challenge to achieve converged $P_{\mathrm{LZ}}$ and $P_{\mathrm{ES}}$ results from numerical accuracy due to the needed very large $t_{\max }$. In the following, only converged results are presented and discussed.

\section{Landau-Zener dynamics}

\subsection{Longitudinal super-Ohmic bath $\Theta=0$}

Figure 1 presents results for $P_{\mathrm{LZ}}$ versus sweep speed for various temperatures for a system-bath coupling strength $\gamma=5 \cdot 10^{-4}$ to a longitudinal super-Ohmic bath. Figure 2, respectively, plots $P_{\mathrm{ES}}$. QUAPI results are given as symbols and lines are NEQB results. Numerical uncertainties are roughly given by the symbol size. QUAPI and NEQB results agree well, thus, justifying the weak coupling NEQB approach. The Landau-Zener probability is not influenced by dissipation at low temperatures $k_{\mathrm{B}} T \lesssim \Delta$ nor for fast drives 


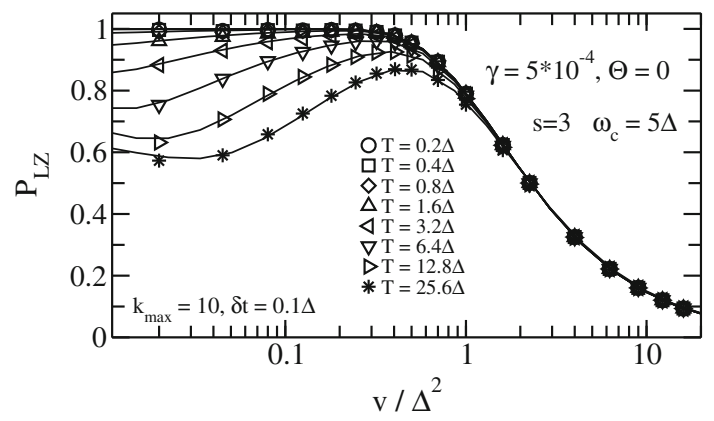

Fig. 1 The Landau-Zener probability $P_{\mathrm{LZ}}$ versus sweep speed for various temperatures at $\omega_{c}=5 \Delta, \gamma=5 \cdot 10^{-4}$, and $\Theta=0$. QUAPI results are given as symbols and lines are NEQB results

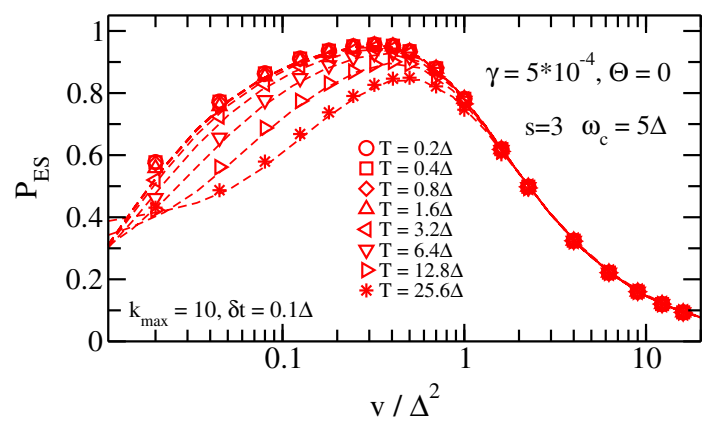

Fig. 2 The excited-state-survival probability $P_{\mathrm{ES}}$ versus sweep speed for various temperatures at $\omega_{c}=5 \Delta, \gamma=$ $5 \cdot 10^{-4}$, and $\Theta=0$. QUAPI results are given as symbols and lines are NEQB results

$v \gtrsim \Delta^{2}$. For $k_{\mathrm{B}} T>\Delta$ and $v<\Delta^{2}$, the LandauZener probability evolves a minimum which deepens with increasing temperature. We observe roughly that the drive speed $v_{\min }$ at the minimum is determined by a condition that the time within the crossing time window equals the (averaged) relaxation time itself similar as for an Ohmic bath [23] but now with extended crossing time window (as discussed below). The picture is that too slow relaxation does not influence the dynamics but too fast relaxation would only bring the system back to the ground state of the TSS whose energy splitting increases continuously after the crossing. The excitedstate-survival probability $P_{\mathrm{ES}}$ is as well only influenced by dissipation in the adiabatic regime, i.e., $v \lesssim \Delta^{2}$ but for all temperatures, since spontaneous emission allows decay of the excited state for any temperature. Decreasing drive speed $v$ gives the system more time close to the avoided crossing and, thus, decreasing $P_{\mathrm{ES}}$. For increasing temperature at a fixed $v$ the excited-state-survival probability, $P_{\mathrm{ES}}$ decreases for $v_{\text {cross }}<v \lesssim \Delta^{2}$, but increases for $v<v_{\text {cross }}$ (best observed in Fig. 4 for larger system-bath coupling $\gamma=5 \cdot 10^{-3}$ ). The drive speed $v_{\text {cross }}$ slightly changes with temperature. Similar qualitative and quantitative results have been obtained for a transversal Ohmic bath [30] at the same systembath coupling strength and for a longitudinal Ohmic bath with $\gamma$ an order of magnitude larger.

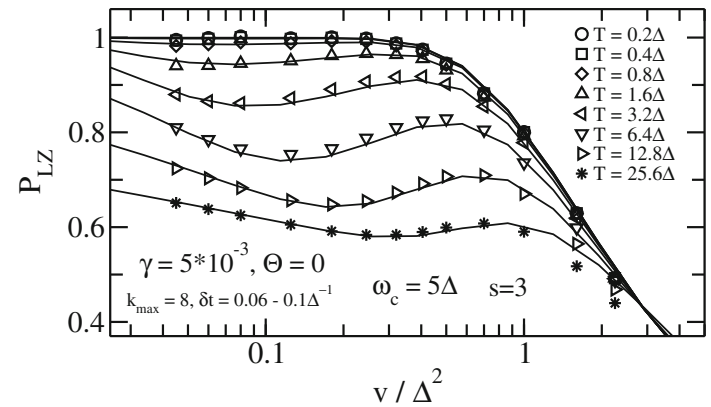

Fig. 3 The Landau-Zener probability $P_{\text {LZ }}$ versus sweep speed for various temperatures at $\omega_{c}=5 \Delta, \gamma=5 \cdot 10^{-3}$ and $\Theta=0$. QUAPI results are given as symbols and lines are NEQB results

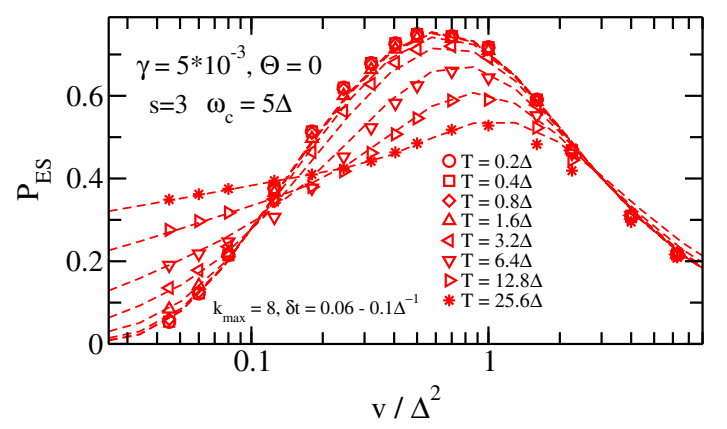

Fig. 4 The excited-state-survival probability $P_{\mathrm{ES}}$ versus sweep speed for various temperatures at $\omega_{c}=5 \Delta, \gamma=$ $5 \cdot 10^{-3}$, and $\Theta=0$. QUAPI results are given as symbols and lines are NEQB results

For a stronger system-bath coupling $\gamma=5 \cdot 10^{-3}$ (see Fig. 3 and 4), QUAPI and NEQB results agree well in the adiabatic regime. For $v \gtrsim \Delta^{2}$ at higher temperatures, $T \gtrsim 3 \Delta / k_{\mathrm{B}}$ NEQB starts overestimating the probabilities $\widetilde{P}_{\mathrm{LZ}}$ and $P_{\mathrm{ES}}$. With increasing systembath coupling, the sweep speed of the minimum $v_{\min }$ is shifted to larger $v$.

For even larger system-bath coupling, QUAPI convergence is hard to achieve within reasonable numerical effort and QUAPI and NEQB differ in the whole range of $v$ values studied. Besides QUAPI convergence with its parameters $k_{\max }$ and $\delta t$, we must ensure convergence of the driving protocol, i.e., in regard to the time window parameter $t_{\max }$ for the simulation running from $-t_{\max } / 2$ to $t_{\max } / 2$. Roughly, in the presented data, $t_{\max }$ varies between $50 \Delta^{-1}$ for $v \gg \Delta^{2}$ to $1500 \Delta^{-1}$ for $v \ll \Delta^{2}$ depending also weakly on temperature. Generally, $P_{\mathrm{ES}}$ needs larger $t_{\max }$ compared to $P_{\mathrm{LZ}}$.

\subsection{Transversal bath $\Theta=\pi / 2$}

In case of a transversal super-Ohmic bath influencing the Landau Zener system, we are only able to achieve convergence for a very small system-bath coupling strength of $\gamma=5 \cdot 10^{-6}$. Figure 5 presents results for $P_{\mathrm{LZ}}$ and $P_{\mathrm{ES}}$ versus sweep speed for various temperatures. QUAPI results are given as symbols and 


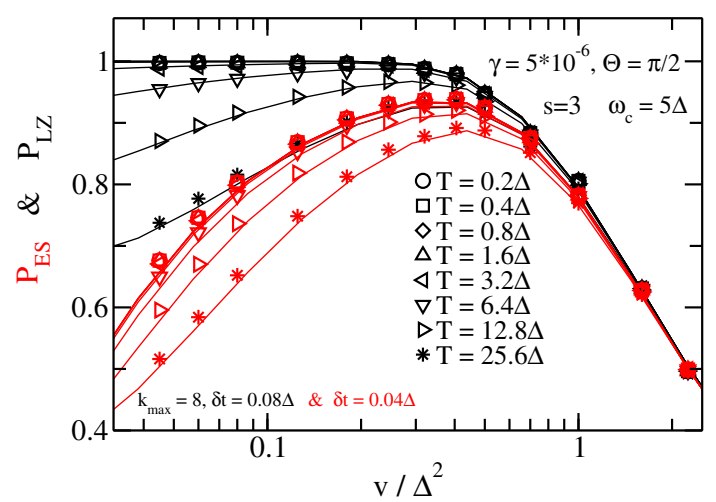

Fig. 5 The Landau-Zener probability $P_{\mathrm{LZ}}$ and the excited-state-survival probability $P_{\mathrm{ES}}$ versus sweep speed for various temperatures at $\omega_{c}=5 \Delta, \gamma=5 \cdot 10^{-6}$ and $\Theta=\pi / 2$. QUAPI results are given as symbols and lines are NEQB results

lines are NEQB results. Both agree well, thus, justifying a weak coupling NEQB approach. Roughly, similar dynamics is observed for a longitudinal bath for coupling strength two orders of magnitude larger.

Converged results for very small sweep speeds $v$ or larger system bath couplings could not be achieved within reasonable numerical effort. In Fig. 5, the minimum in $P_{\mathrm{LZ}}$ is not resolved, but will be found at smaller sweep speeds according to the easily obtained NEQB results. Since a minimum at smaller sweep speed $v$ reflects a smaller relaxation rate $[22,23]$, this points towards a situation where convergence is not hindered by long time memory due to strong system-bath coupling but due to very long needed simulation times to capture all bath effects. This hints at a very extended crossing time window for the transversal super-Ohmic environment and is backed by the observation that convergence needs larger $t_{\max }$ compared to the case of a longitudinal super-Ohmic bath.

We also simulated the time evolution of the driven TSS, i.e., $\operatorname{Tr}\{\hat{g} \rho(t)\}$ and $\operatorname{Tr}\{\hat{e} \rho(t)\}$ for smaller $t_{\max }$ and find very good agreement between NEBQ and QUAPI results. The final $\operatorname{Tr}\left\{\hat{g} \rho\left(t_{\max }\right)\right\}$ and $\operatorname{Tr}\left\{\hat{g} \rho\left(t_{\max }\right)\right\}$; however, depend strongly on $t_{\max }$ and, thus, this does not allow to determine $P_{\mathrm{LZ}}$ and $P_{\mathrm{ES}}$. When increasing $t_{\mathrm{max}}$, the accumulated numerical error in QUAPI deteriorates the according simulation. This numerical error due to very long simulation times is easily avoided in NEBQ and, thus, we believe the NEBQ results to be reliable.

\subsection{Mixed bath $-\pi / 2<\Theta \leq \pi / 2$}

To complete our investigation of the Landau Zener dynamics in a super-Ohmic environment, we have additionally studied mixed bath cases, i.e., a single bath which exhibits both longitudinal and transversal coupling with $-\pi / 2<\Theta \leq \pi / 2$. For an Ohmic bath, it was observed that small transversal contributions to a mainly longitudinal bath shift the minimum in $P_{\mathrm{LZ}}$, i.e., $v_{\mathrm{LZ}}$, depending on the sign of the angle $\Theta$. Arising from

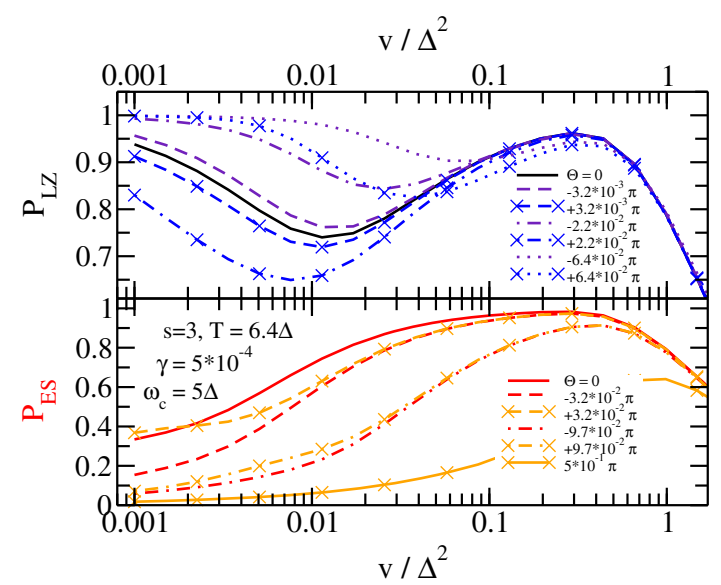

Fig. 6 The Landau-Zener probability $P_{\mathrm{LZ}}$ (upper figure) and the excited-state survival probability $P_{\mathrm{ES}}$ (lower figure) versus sweep speed for various mixing angles $-\pi / 2<\Theta \leq$ $\pi / 2$ at fixed temperature $T=6.4 \Delta / k_{\mathrm{B}}$ and $\omega_{c}=5 \Delta$ and $\gamma=5 \cdot 10^{-4}$. Only NEQB results are shown. Symbols are used to differentiate curves

this asymmetry, an environmental rocking ratchet can be designed which allows rectification in energy transport through an according system [48].

Figure 6 presents the Landau Zener probability (upper figure) and the excited-state survival probability (lower figure) versus drive speed $v$ for various mixing angles $\Theta$, at fixed temperature $T=6.4 \Delta / k_{\mathrm{B}}$ and a system-bath coupling $\gamma=5 \cdot 10^{-4}$. Only NEBQ results are shown, since due to the transversal super-Ohmic bath contribution converged QUAPI results could not be achieved. As pointed out above, NEBQ results are believed to be fully reliable at the studied couplings. For the Landau Zener probability, we observe strong differences depending on the sign of $\Theta$. The effect is limited to a small range of angles, i.e. $|\Theta| \lesssim \pi / 10$, but is quantitatively substantial; for example, $P_{L Z}(\Theta=$ $\left.2.2 \cdot 10^{-2} \pi\right)-P_{L Z}\left(\Theta=-2.2 \cdot 10^{-2} \pi\right) \gtrsim 0.2$. Slightly smaller differences are found in the same limited angle range for the excited-state survival probability. These differences are present generally at smaller sweep speeds compared to the Landau-Zener probability.

\section{Crossing time window}

Finding converged numerical results for the LandauZener dynamics (as presented in the previous section) shows two important points. First, convergence regarding the protocol simulation time $t_{\max }$ highlights that environmental influence acts only in a finite time window around the avoided crossing at $t=0$. Second, we determine system-bath coupling strength regimes where the NEQB accurately describe the dynamics. This allows us to rationalize the crossing time window by the time (driving)-dependent relaxation rate of the NEQB. Importantly, it also shows that pure dephasing 
of the super-Ohmic environment seems to have no significant influence on the studied Landau-Zener dynamics.

Figure7 plots the relaxation rate $\Gamma_{1}[s, \Theta](t)$ [see Eq. (8)] for longitudinal (star and triangle symbols) and transversal (cross and square symbols) Ohmic (square and triangle symbols) as well as super-Ohmic (cross and star symbols) baths. For a longitudinal Ohmic bath (magenta triangles), the relaxation rate is maximal at the avoided crossing $t=0$ and decreases strongly on both sides of the crossing with $\propto \exp \left(-E(t) / \omega_{c}\right) / E(t)$. For a transversal Ohmic bath, the relaxation rate vanishes at $t=0$, but quickly increases away from the crossing due to $\propto(v t)^{2} \exp \left(-E(t) / \omega_{c}\right) / E(t)$. Finally, the cut-off frequency of the environment strongly suppresses relaxation further away from the crossing. A longitudinal super-Ohmic bath exhibits a similar relaxation rate $\propto E(t) \exp \left(-E(t) / \omega_{c}\right)$ away from the crossing (where $E(t) \simeq(v t)$ ), but in contrast does not vanish at the crossing. A transversal super-Ohmic bath leads to a vanishing relaxation rate at $t=0$, but increases even stronger away from the crossing, i.e., $\propto$ $(v t)^{2} E(t) \exp \left(-E(t) / \omega_{c}\right)$. Despite the large differences of the time-dependent relaxation rate in these four bath cases, convergence in all cases is finally ensured by the cut-off frequency of the bath. Generally, for a bath with spectral exponent $s$, the relaxation rate (see Eq. (8) ) is $\propto(E(t))^{s-2} \exp \left(-E(t) / \omega_{c}\right)$ for a longitudinal and $\propto(v t)^{2}(E(t))^{s-2} \exp \left(-E(t) / \omega_{c}\right)$ for a transversal bath. With increasing $s>0$ for a transversal bath and $s>2$ for a longitudinal bath two peaks symmetric around $t=0$ are present whose heights and widths increase with $s$. For a longitudinal bath with $0<s<2$, a single peak centered at $t=0$ is present whose widths increase with $s$. The crossing time window increases accordingly with increasing $s$.

The quantitative differences for the various bath cases and, accordingly, the relevant time windows in which relaxation will influence the Landau-Zener dynamics dominantly, can be estimated from Fig.7. In all cases, the time window is centered around the avoided crossing, but only for a longitudinal Ohmic bath, it actually peaks at the crossing. The relaxation rate in the other three cases has its peak when the energy splitting of the TSS equals for $s=1$ with $\Theta=\pi / 2$ and $s=3$ with $\Theta=0$ roughly five times and for $s=3$ with $\Theta=\pi / 215$ times the bath cut-off energy. To compare the overall influence of relaxation in the four cases, we determine

$$
\Xi[s, \Theta]=\int_{-\infty}^{\infty} d t \Gamma_{1}[s, \Theta](t)
$$

and find $\Xi[1,0]=3.5 \Delta^{2} /(v \gamma)$ for longitudinal Ohmic relaxation, $\Xi[1, \pi / 2]=47.8 \Delta^{2} /(v \gamma)$ for transversal Ohmic relaxation, $\Xi[3,0]=51.3 \Delta^{2} /(v \gamma)$ for longitudinal super-Ohmic relaxation and $\Xi[3, \pi / 2]=$ $7475 \Delta^{2} /(v \gamma)$ for transversal super-Ohmic relaxation. The ratios of these values reflect nicely the necessary ratios of system-bath coupling strengths to obtain sim-

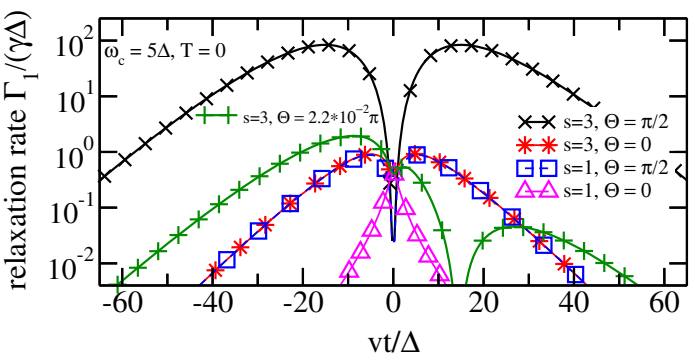

Fig. 7 The relaxation rate $\Gamma_{1}[s, \Theta](t)$ (in units of $\gamma \Delta$ ) versus time at zero temperature for Ohmic and super-Ohmic longitudinal and transversal baths

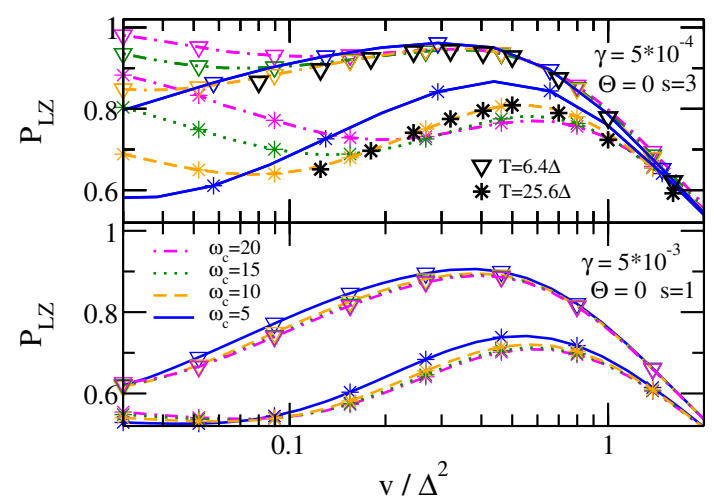

Fig. 8 The Landau-Zener probability $P_{\mathrm{LZ}}$ versus sweep speed for two temperatures $T=6.4 \Delta k_{\mathrm{B}}$ and $T=25.6 \Delta / k_{\mathrm{B}}$, various bath cut-off frequencies $\omega_{c}$ and (upper figure) a longitudinal super-Ohmic bath with $\gamma=5 \cdot 10^{-4}$ and (lower figure) a longitudinal Ohmic bath with $\gamma=5 \cdot 10^{-3}$. Black symbols are QUAPI data. All other data are NEQB results

ilar Landau-Zener dynamics (similar values for $v_{\min }$ ) for the different bath cases.

The vastly different crossing time windows depend in all cases strongly on $\omega_{c}$ except for a longitudinal Ohmic bath. This is reflected in a strong dependence of the Landau-Zener dynamics at $\omega_{c}$. Figure 8 plots the Landau-Zener probability $P_{\mathrm{LZ}}$ versus sweep speed at two temperatures $T=6.4 \Delta k_{\mathrm{B}}$ and $T=25.6 \Delta / k_{\mathrm{B}}$. The upper figure represents data of a longitudinal superOhmic bath with $\gamma=5 \cdot 10^{-4}$ for four different cut-off frequencies $\omega_{c}$. The black symbols are QUAPI data for $\omega_{c}=10 \Delta$. All other data are determined by NEQB. Clearly, a very strong dependence on $\omega_{c}$ is observed within the adiabatic regime $v \lesssim \Delta^{2}$. In contrast, a longitudinal Ohmic bath (data depicted for $\gamma=5 \cdot 10^{-3}$ in the lower figure of Fig. 8) shows only a very weak dependence on $\omega_{c}$.

For mixed bath cases, the crossing time window is not symmetric around $t=0$ anymore. The green +symbols in Fig.7 plot the relaxation rate $\Gamma_{1}[s, \Theta=$ $\left.2.210^{-2} \pi\right](t)$ for a super-Ohmic bath. Clearly, an asymmetric behaviour is observed with a dominant peak for $t<0$. Changing the sign of $\Theta$ results in a curve mirrored at the $t=0$ axis (not shown in Fig. 7). A peak 
in the relaxation rate for $t<0$ results in strong relaxation, while the energy splitting is still getting smaller and thermal excitations become more likely. Thus, one expects a deeper minimum in $P_{L Z}$ as observed. In contrast, a peak at positive times reflects relaxation being dominant, while the energy splitting is getting larger again and thermal excitations are freezing out. Then, the relaxation back to the ground state is more profound and, thus, a shallower minimum in $P_{L Z}$ results.

\section{Conclusion}

We studied the Landau-Zener dynamics, i.e., the Landau-Zener probability and the excited-statesurvival probability, under the influence of an superOhmic environment to reveal whether its influence is restricted to a crossing time window around the avoided crossing and, if so, what determines this crossing time window. Previously, for an Ohmic environment, it has been observed that the bath influence was restricted to such a crossing window. For a longitudinal Ohmic bath, this crossing time window is determined by the driven quantum system, i.e., the tunnel coupling between the driven states and the driving speed. A transversal Ohmic bath, in contrast, influences the dynamics in a much wider time window around the avoided crossing determined by temperature and the environmental spectrum. Specifically, the influence is limited to the times where the bath cut-off energy (or frequency) exceeds the energy splitting in the quantum system and, accordingly, energy exchange between both is possible by a single-boson process.

We have here determined the Landau-Zener and the excited-state survival probabilities numerically exact employing the quasi-adiabatic path integral and within a Markovian weak coupling approach. As a first result, we find converged results with increasing simulation times which highlights that the Landau-Zener dynamics under the influence of a super-Ohmic environment is still focussed within a crossing time window around the avoided crossing. A second result is that for weak coupling, both approaches yield identical results. This shows that pure dephasing does not significantly influence the studied dynamics, since super-Ohmic pure dephasing is non-Markovian and, thus, not taken into account in a Markovian weak coupling approach.

Then, we employ the Markovian weak coupling approach, i.e., the time-dependent effective dissipation rates, i.e., relaxation and according decoherence rates, to rationalize and concretize the crossing time window. The relaxation rate exhibits maximal value during the driving when the systems' energy splitting is roughly five times the bath cut-off energy for a longitudinal and 15 times for a transversal super-Ohmic environment. The according time windows between the two maximal values exhibit the crossing time windows. We observe also that a longitudinal super-Ohmic bath shows a crossing time window similar to a transversal Ohmic bath.
Finite crossing time windows are essential to employ simple qualitative results from the Landau-Zener model to interpret according experimental results. While we have seen that the Landau-Zener probability and the excited-state-survival probability are both little influenced by pure dephasing contributions from our studied environments, we would expect that other observables at an avoided crossing might be strongly influenced by dephasing, for example, when using an avoided crossing as a 'beam splitter'. Then, no finite crossing time window would be expected and all results might strongly depend on the driving protocol away from the avoided crossing.

The extended crossing time window for the superOhmic spectra restricted our investigation to very weak system-bath couplings. Intermediate-to-strong couplings allow multiple-boson processes between system and environment which would extend the crossing time windows even further. While a longitudinal Ohmic bath hardly influences the non-adiabatic regime of the Landau-Zener dynamics, the extended crossing time windows for super-Ohmic bath might allow a strongly bath-influenced non-adiabatic dynamics not observed so far.

Funding Open Access funding enabled and organized by Projekt DEAL.

Data Availability Statement This manuscript has no associated data or the data will not be deposited. [Authors' comment: The datasets generated during and/or analysed during the current study are available from the corresponding author on reasonable request.]

Open Access This article is licensed under a Creative Commons Attribution 4.0 International License, which permits use, sharing, adaptation, distribution and reproduction in any medium or format, as long as you give appropriate credit to the original author(s) and the source, provide a link to the Creative Commons licence, and indicate if changes were made. The images or other third party material in this article are included in the article's Creative Commons licence, unless indicated otherwise in a credit line to the material. If material is not included in the article's Creative Commons licence and your intended use is not permitted by statutory regulation or exceeds the permitted use, you will need to obtain permission directly from the copyright holder. To view a copy of this licence, visit http://creativecomm ons.org/licenses/by/4.0/.

\section{References}

1. L.D. Landau, Phys. Z. Sowjetunion 2, 46 (1932)

2. C. Zener, Proc. R. Soc. Lond. A 137, 696 (1932)

3. E. Majorana, Nuovo Cimento 9, 43 (1932)

4. E.C.G. Stueckelberg, Helv. Phys. Acta 5, 369 (1932)

5. A. Nitzan, Chemical Dynamics in Condensed Phases (Oxford UP, Oxford, 2006)

6. W. Wernsdorfer, R. Sessoli, Science 284, 133 (1999)

7. D. Rosenberg, P. Nalbach, D.D. Osheroff, Phys. Rev. Lett. 90, 195501 (2003) 
8. S. Ludwig, P. Nalbach, D. Rosenberg, D. Osheroff, Phys. Rev. Lett. 90, 105501 (2003)

9. P. Nalbach, D.D. Osheroff, S. Ludwig, J. Low Temp. Phys. 137, 395 (2004)

10. P. Nalbach, Phys. Rev. B 71, 052201 (2005)

11. J.R. Petta, H. Lu, A.C. Gossard, Science 327, 669 (2010)

12. K.D. Petersson, J.R. Petta, H. Lu, A.C. Gossard, Phys. Rev. Lett. 105, 246804 (2010)

13. T. Fujita, T.A. Baart, C. Reichl, W. Wegscheider, L.M.K. Vandersypen, npj Quant. Inf. 3 (2017)

14. A.R. Mills, D.M. Zajac, M.J. Gullans, F.J. Schupp, T.M. Hazard, J.R. Petta, Nat. Commun. 10 (2019)

15. F. Ginzel, A.R. Mills, J.R. Petta, G. Burkard, Phys. Rev. B 102, 195418 (2020)

16. C.J. van Diepen, T.K. Hsiao, U. Mukhopadhyay, C. Reichl, W. Wegscheider, L.M.K. Vandersypen, Nat. Commun. 12 (2021)

17. J. Yoneda, W. Huang, M. Feng, C.H. Yang, K.W. Chan, T. Tanttu, W. Gilbert, R.C.C. Leon, F.E. Hudson, K.M. Itoh et al., Nat. Commun. 12 (2021)

18. J.A. Krzywda, L Cywiński, Phys. Rev. B 101, 035303 (2020)

19. J.A. Krzywda, L Cywiński, Phys. Rev. B 104, 075439 (2021)

20. U. Weiss, Quantum Dissipative Systems, 2nd edn. (World Scientific, Singapore, 1998)

21. A.J. Leggett, S. Chakravarty, A.T. Dorsey, M.P.A. Fisher, A. Garg, W. Zwerger, Rev. Mod. Phys. 59, 1 (1987)

22. P. Nalbach, M. Thorwart, Phys. Rev. Lett. 103, 220401 (2009)

23. P. Nalbach, M. Thorwart, Chem. Phys. 375, 234 (2010)

24. Y. Kayanuma, J. Phys. Soc. Jpn. 53, 108 (1984)

25. Y. Kayanuma, J. Phys. Soc. Jpn. 53, 118 (1984)

26. P. Ao, J. Rammer, Phys. Rev. Lett. 62, 3004 (1989)

27. Y. Kayanuma, H. Nakayama, Phys. Rev. B 57, 13099 (1998)
28. M. Wubs, K. Saito, S. Kohler, P. Hänggi, Y. Kayanuma, Phys. Rev. Lett. 97, 200404 (2006)

29. V.L. Pokrovsky, D. Sun, Phys. Rev. B 76, 024310 (2007)

30. S. Javanbakht, P. Nalbach, M. Thorwart, Phys. Rev. A 91, $052103(2015)$

31. R.S. Whitney, M. Clusel, T. Ziman, Phys. Rev. Lett. 107, 210402 (2011)

32. Z. Huang, Y. Zhao, Phys. Rev. A 97, 013803 (2018)

33. R.K. Malla, M.E. Raikh, Phys. Rev. B 97, 035428 (2018)

34. R. Chen, Phys. Rev. B 101, 125426 (2020)

35. P. Nalbach, J. Knörzer, S. Ludwig, Phys. Rev. B 87, 165425 (2013)

36. P. Nalbach, Phys. Rev. A 90, 042112 (2014)

37. N. Makri, D.E. Makarov, J. Chem. Phys. 102, 4600 (1995)

38. N. Makri, D.E. Makarov, J. Chem. Phys. 102, 4611 (1995)

39. M. Thorwart, P. Reimann, P. Hänggi, Phys. Rev. E 62, 5808 (2000)

40. T. Palm, P. Nalbach, J. Chem. Phys. 149, 214103 (2018). https://doi.org/10.1063/1.5051652

41. A. Würger, Phys. Rev. Lett. 78, 1759 (1997)

42. A. Würger, Phys. Rev. B 57, 347 (1998)

43. P. Nalbach, Phys. Rev. B 66, 134107 (2002)

44. L. Hartmann, I. Goychuk, M. Grifoni, P. Hänggi, Phys. Rev. E 61, R4687 (2000)

45. P. Solinas, M. Möttönen, J. Salmilehto, J.P. Pekola, Phys. Rev. B 82, 134517 (2010)

46. L. Arceci, S. Barbarino, R. Fazio, G.E. Santoro, Phys. Rev. B 96, 054301 (2017)

47. L. Arceci, S. Barbarino, R. Fazio, G.E. Santoro, Phys. Rev. B 98, 019902 (2018)

48. P. Nalbach, N. Klinkenberg, T. Palm, N. Müller, Phys. Rev. E 96, 042134 (2017) 